\section{Drug therapy in older people with heart failure}

Editor - We read with interest the review article by Mukhtar and Jackson entitled Drug therapy in older adults (part 2). ${ }^{1}$ In the article the authors provide an overview on the drug treatment of heart failure in older patients. However, we were surprised that digoxin, one of the oldest drugs in the management of heart failure was not mentioned as a treatment option. Clinical evidence is in favour of digoxin in diastolic heart failure as well as systolic heart failure through improvement of early diastolic function and possible reduction in sympathetic humoral activity.

The benefits are not restricted to patients with heart failure in atrial fibrillation but also in sinus rhythm. In a systematic review of 13 trials which included over 7,000 patients with heart failure in sinus rhythm, digitalis had a positive effect on improving symptoms and reducing hospitalisation. ${ }^{2}$

The earlier Digitalis Investigation Group trial had also showed, in addition to $6 \%$ less hospitalisation in the treatment, a trend toward a beneficial effect of digoxin in pump failure death, but this was offset by mortality from non-cardiovascular causes ${ }^{3}$.

Therefore in older patients with moderately severe heart failure who remain symptomatic despite optimum treatment with diuretics, angiotensin-converting-enzyme inhibitors and beta blockers, it is worthwhile considering the addition of digoxin. It is important to point out that the benefits of digoxin occur with low maintenance dosage which is usually between $62.5-125 \mu \mathrm{g}$ daily.

The benefits of digoxin mentioned above cannot be overemphasised in the vast majority of the older frailer population with heart failure. This is important as the NHS is aiming towards keeping the frail elderly in their own environment for as long as possible rather than hospitalisation. Clinicians working in acute frailty units are likely to come across more of these patients and can best serve this cohort of patients well by asking why they cannot be on digoxin. Every little helps especially on a frailty ward!

\section{References}

1 Mukhtar O and Jackson S. Drug therapies in older adults (part 2). Clin Med 2015;15:155-9.

2 Hood WB, Dans AL, Guyatt GH et al. Digitalis for treatment of congestive heart failure in patients in sinus rhythm: a systematic review and meta-analysis. J Card Fail 2004;10:155-164.

3 The Digitalis Investigation Group. The effect of digoxin on mortality and morbidity in patients with heart failure. $N$ Engl J Med 1997;336:525-33.

GREGORY BABALOLA Princess Royal University Hospital, Orpington, UK

AZA ABDULLA

Princess Royal University Hospital, Orpington, UK

\section{Response}

Editor - We thank the correspondents for their letter in response to our recent article 'Drug therapies in older adults (part 2)'. ${ }^{1}$ While digoxin is well established in the management of older patients with atrial fibrillation with or without heart failure, the evidence base for digoxin in frail older patients is not established.

The Digitalis Investigation Group study referred to in your letter enrolled 6,800 patients with documented left ventricular ejection fractions of $45 \%$ or less. ${ }^{2}$ We are concerned that in this study, as the serum digoxin concentration increased, the absolute risk in mortality rose versus patients receiving placebo. In such patients the absolute mortality rate was $11.8 \%(95 \%$ confidence interval $5.7-18.0 \% ; \mathrm{p}<0.001)$ higher than those receiving placebo. The effect of frailty on this association is unknown.

We included the section on neprilysin inhibitors to establish that there is a pipeline of newer agents, but we made the point that their role is not established. We agree, however, that we should have included digoxin here discussing its newer roles rather than the indication established described by Sir William Withering. Clinicians managing frailty units should restrict their therapeutic choices to those that have an evidence base in frail patients, or where clinical circumstances are in favour of extrapolating from fitter populations.

OMAR MUKHTAR

$S p R$ in clinical pharmacology and therapeutics, King's Health Partners, King's College Hospital, London, UK

STEPHEN HD JACKSON Professor of clinical gerontology, Department of Clinical Gerontology, King's Health Partners, King's College Hospital, London, UK

\section{References}

1. Mukhtar O, Jackson SH. Drug therapies in older adults (part 2). Clin Med 2015; 15:155-9.

2. The Digitalis Investigation Group. The effect of digoxin on mortality and morbidity in patients with heart failure. $N$ Engl J Med 1997;336:525-33.

\section{A cough that doesn't fit the mould}

Editor - We read with interest the lesson of month entitled $A$ cough that doesn't fit the mould (Clin Med 2015;15:492-4). This is an interesting case report, which we believe highlights the difficulty in making a diagnosis and classifying Aspergillusrelated pulmonary disease. Baggott et al have made a diagnosis of Aspergillus tracheobronchitis and mention that it is part of the spectrum of invasive Aspergillus disease. We believe it would be worth adding that both saprophytic (non-invasive) and invasive forms of Aspergillus tracheobronchitis exist. ${ }^{1,2}$ The latter requires the demonstration of fungal hyphae invading the bronchial mucosa, which was not demonstrated in this case. Where fungal hyphae are found in the bronchial tree without invasion this could be due to obstructing bronchial aspergillosis, which requires the absence of allergic disease, ${ }^{1}$ or mucoid impaction that is a non-specific sign of Aspergillus bronchial disease and is also a feature of allergic bronchopulmonary aspergillosis (ABPA). The case presented by Baggott et al had mucoid impaction resulting in obstruction and collapse of the right middle lobe. However, the patient developed features of an allergic reaction to Aspergillus evidenced by the positive gradeIII specific Aspergillus IgE, high total IgE level (1,519 kU/L) and 